\title{
LLNL Medical and Industrial Laser Isotope Separation: Large Volume, Low Cost Production Through Advanced Laser Technologies
}

\author{
K. Scheibner \\ B. Comaskey \\ M. Shaw \\ J. Wilder
}

September 2, 1998

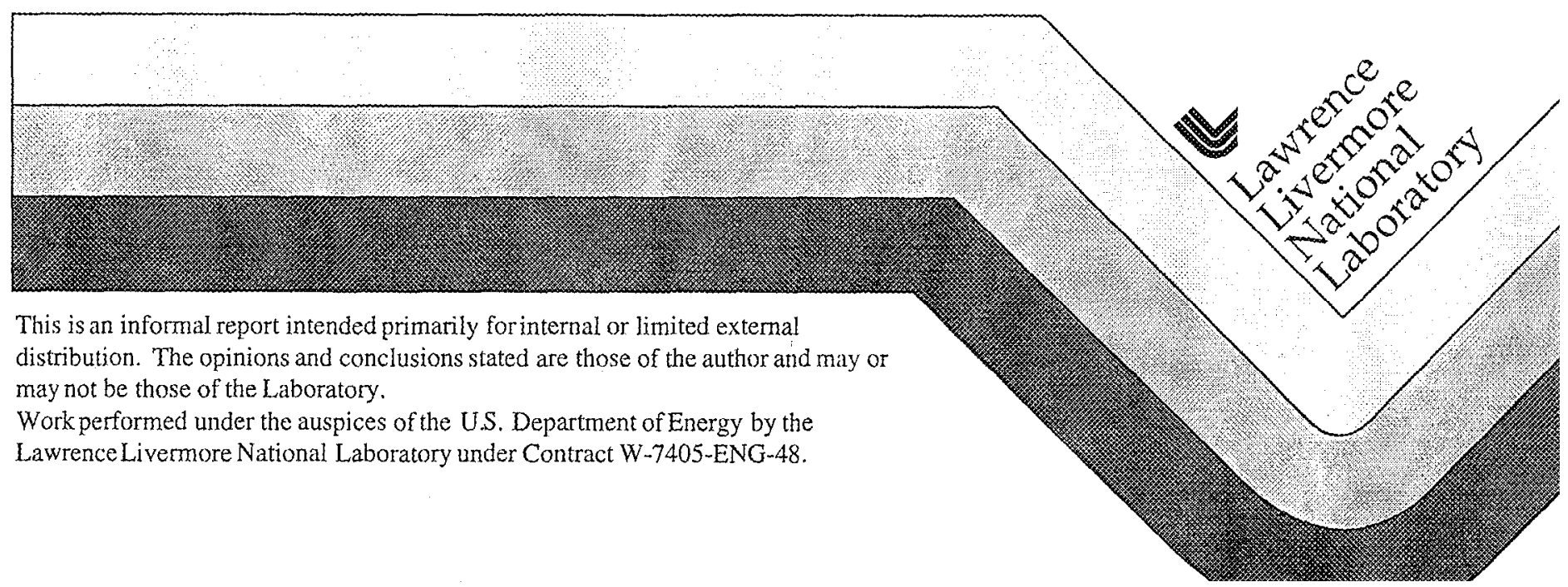




\section{DISCLAIMER}

This document was prepared as an account of work sponsored by an agency of the United States Government. Neither the United States Government nor the University of California nor any of their employees, makes any warranty, express or implied, or assumes any legal liability or responsibility for the accuracy, completeness, or usefulness of any information, apparatus, product, or process disclosed, or represents that its use would not infringe privately owned rights. Reference herein to any specific commercial product, process, or service by trade name, trademark, manufacturer, or otherwise, does not necessarily constitute or imply its endorsement, recommendation, or favoring by the United States Government or the University of California. The views and opinions of authors expressed herein do not necessarily state or reflect those of the United States Government or the University of California, and shall not be used for advertising or product endorsement purposes.

This report has been reproduced directly from the best available copy.

Available to DOE and DOE contractors from the Office of Scientific and Technical Information

P.O. Box 62, Oak Ridge, TN 37831

Prices available from (615) 576-8401, FTS 626-8401

Available to the public from the

National Technical Information Service

U.S. Department of Commerce

5285 Port Royal Rd.,

Springfield, VA 22161 


\section{LLNL Medical and Industrial Laser Isotope Separation: Large Volume, Low Cost Production Throngh Advanced Laser Technologies}

Principal Investigator:

Co-Investigators:

Responsible Associate Director:
Karl Scheibner

Atomic Vapor Laser Isotope Separation Program

Brian Comaskey, Michael Shaw, and John Wilder Atomic Vapor Laser Isotope Separation Program

E. Michael Campbell

Laser Directorate

Work performed under the auspices of the U.S. Department of Energy by Lawrence Livermore National Laboratory under Contract W-7405-Eng-48; and was sponsored by the United States Enrichment Corporation. 


\section{LLNL Medical and Industrial Laser Isotope Separation: Large Volume, Low Cost · Production Through Advanced Laser Technologies}

\section{Project Concept and Goal}

The goal of this LDRD project was to demonstrate the technical and economical feasibility of applying laser isotope separation technology to the commercial enrichment $(>1 \mathrm{~kg} / \mathrm{y})$ of stable isotopes. A successful demonstration would well position the laboratory to make a credible case for the creation of an ongoing medical and industrial isotope production and development program at LLNL. Such a program would establish LLNL as a center for advanced medical isotope production, successfully leveraging previous LLNL Research and Development hardware, facilities, and knowledge.

There were several reasons that made this project timely and appropriate for LLNL LDRD funding. First, several of the major users from the medical and industrial communities had expressed interest in lower prices (compared to calutrons for metal isotopes, cryogenic distillation for carbon and oxygen isotopes), and a reliable, domestic source (some isotopes only available from Russia). Second, DOE announced in December 1993 its intention to get back into isotope production, through the Office of Isotope Production and Distribution, to "maintain an indigenous U.S. source of isotopes essential for health care, research, and industrial applications". This reversal in its previous "income-neutral" policy was driven in large part by the critical needs of the medical and biotechnological communities. Furthermore, this policy change was an implicit recognition, both within and outside government, of the vital role isotopes have played in medical advances, and are expected to continue to play in the future.

Third, new medical health technologies have led to increased consideration of the usage of many new isotopes. Recent examples include strontium, barium, calcium, samarium, molybdenum, cadmium, and iron. Many of these potential applications suffer from the prohibitively expensive cost of enriched (or purified) isotopes, where the price usually scales as $\$ 1 / \mathrm{mg} / \mathrm{X}_{\mathrm{F}}$ (where $\mathrm{X}_{\mathrm{F}}$ is the natural abundance of the isotope of interest). Thus, for many isotopes, even the cost of feasibility studies can be prohibitive.

Fourth, for those atomic species and isotopes for which laser isotope separation (LIS) is technically possible LIS has three distinct advantages: a) Minor isotopes can be enriched or stripped with good single-step selectivity; b) The laser powers needed at the required wavelengths are consistent with current Laboratory capabilities, and translate into high throughputs that are economically attractive for applications that require $\geq 100 \mathrm{~g} / \mathrm{y}$ product; and c) For those isotopes it can access, and for large enough demand, LIS should be the least expensive production technique. 


\section{Thallium-203 as a Case Study}

Initially, thallium provided a useful case study. It has two naturally occurring isotopes, ${ }^{203} \mathrm{~T} 1(30 \%)$ and ${ }^{205} \mathrm{~T} 1(70 \%) .{ }^{203} \mathrm{~T} 1$ is a precursor to ${ }^{201} \mathrm{~T} 1$ that is widely used for cardiological and cerebral imaging. It is consumed at a rate of about 3 kilograms per year, corresponding to about $\$ 7 \mathrm{M} / \mathrm{y}$ (currently produced by calutrons). The initial set of laser wavelengths and power requirements for laser isotope separation thought to be applicable at the outset of the project did not present huge technical hurdles for isotopic enrichment, and the vaporization temperature required for reasonable throughput rates looked to be obtainable with a thermal source. One of the lasers needed for thallium enrichment was anticipated to be a titanium-sapphire (Ti:S) laser, pumped by a neodymium yttria aluminum garnet (Nd:YAG) laser, both lasers that the uranium Atomic Vapor Laser Isotope Separation (AVLIS) Program has had significant experience with over the last 15 years. The Ti:S laser, because it is tunable, can be applied to a number of other isotopes of medical and industrial interest. Because a market already existed and because at least two companies had expressed interest in this isotope, a successful $\mathrm{Tl}$ enrichment campaign would provide credibility for further medical isotope missions.

The economics of laser isotope separation of $\mathrm{T} 1$ looked promising. As discussed below, the unit price of enriched ${ }^{203} \mathrm{~T} 1$ could be lower than $\$ 1,000 / \mathrm{g}$ for a system capable of producing $>2 \mathrm{~kg} / \mathrm{y}$ (minor modifications of this same system could lead to considerably larger throughputs). This is to be compared with the calutron's price of $\$ 2,000-\$ 3,000 / \mathrm{g}$.

The calutron's great strength is its flexibility, and its application to those isotopes required in small amounts at high purity. By contrast, laser isotope separation requires examination of each atomic species in consideration and subsequent and substantial system modifications. For those cases that appear promising, development costs and capital costs of laser and separator hardware will dominate. For large enough throughputs $(\geq 100 \mathrm{~g} / \mathrm{y})$, however, unit costs are likely to be less than the calutrons. Because of the high specificity of the laserbased process, enrichment costs depend on product rate, not feed rate. Furthermore, emerging solid-state laser technologies present opportunities for decreasing these costs.

\section{Laboratory Preparation and Initial Results}

From the outset it was clear that a separator area and a laser area would be necessary to allow the co-development of the two individual technologies. Once a laboratory was identified, equipment hardware was found and secured. A nearly complete separator vacuum chamber with all the required process laser ports was found, along with most of a functioning control system. The separator system neatly fit on an accompanying glovebox that was also necessary for safe handling of toxic thallium. All of this hardware was moved into the facility (see Figs. 1a-d). At the same time, heater power supplies required for the thermal source and an extractor power supply were also found and transferred to the facility. 
The initial separator development focused on the vaporizer. Because of the care with which thallium needs to be handled (the toxicity is comparable to arsenic), zinc was initially used as a surrogate because of both its ease of vaporization and its benign ES\&H characteristics. Using zinc, a number of different vaporizers were conceived, built, and tested. The requirement was a vapor source that could vaporize $>10$ grams per hour into a vacuum with well collimated (high Mach number, low temperature) flow characteristics. Several generations of vaporizers were developed, none completely satisfactory, but each generation more complicated than the previous generation. A new strategy was developed and implemented that, over the course of a year, eventually lead to a satisfactory source.

This particular facility was chosen because of the close proximity of sufficient monolith space near the separator system. Monoliths are typically required for LIS applications because of the frequency stability requirements, usually less than $100 \mathrm{MHz}$. At the same time that the separator system was being built up, a parallel effort was undertaken to design, procure, assemble, and test the laser system, one component of which required Ti:S as the tunable medium. Ti:S is tunable over the range of $650 \mathrm{~nm}$ to about $920 \mathrm{~nm}$ (see Fig. 2). This effort was set up in the laser development laboratory (see Fig. 3), in close proximity to the cryostatic amplifier. In addition to the oscillator and the amplifier, laser hardware for the remaining process colors (a combination of solid state lasers and dye lasers) needed to be specified, designed, components ordered, assembled, and developed into functioning systems. All of this work was also carried out in the laser development laboratory.

The specific wavelengths used for any laser isotope separation scheme depends on the unique absorption resonance frequencies of the atom under consideration. These frequencies are usually well known, at least for the lowest lying levels. However, to adequately specify the laser system needed to efficiently and selectively ionize the isotope of interest, more state information is required. The most important includes the absorption cross section (required to determine the minimum laser pulse fluence requircments), isotope shifts for all the transitions (an important parameter since it can place limitations on the maximum allowable Doppler width of the atomic vapor as well as the optical specificity of the process), and the nuclear hyperfine structure (needed as part of the selective photoionization scheme). The project required an active spectroscopic investigation to supplement the published data on thallium for these measurements.

In Fig. 4, it is schematically shown what had been known of the relevant spectroscopic parameters and the initially-intended photoionization scheme: four optically coupled bound states, forming a closed system with respect to the ionization channel. The first excited state has a short lifetime (compared to laser pulse lengths), and decays with nearly equal probability to the ground and metastable states. The photoabsorption cross sections connecting these three levels are very large and only require a few watts to statistically populate them. An additional development laser was proposed to couple in a fourth metastable state. These four levels, when statistically populated by the three lasers, will act essentially as a single level with respect to the photoionization process. Furthermore, the selectivity of the lasers makes possible the excitation of the first and second excited states of the ${ }^{203} \mathrm{~T} 1$ only. For this ionization pathway the 
wavelength connecting the second excited state and the continuum must be less than $1.26 \mu$. The necessary laser power to saturate the ionization channel is proportional to the photoionization cross section. Part of the efforts of this proposal was to search for autoionization resonances consistent with the available wavelength region. For the purpose of estimating the production cost a cross section of $\sigma=5 \times 10^{-17} \mathrm{~cm}^{2}$ was assumed, not atypical of non-resonant cross sections. The corresponding saturation laser power is about $150 \mathrm{~W}$.

Early into the spectroscopic investigation it was recognized that this photoionization scheme would prove to be cost prohibitive, and would be very difficult to implement. An alternate photoionization scheme was proposed, and the technical validity was verified in the spectroscopy laboratory. Shown in Figs. $5 a$ and $5 b$ is the Spectroscopy Laboratory used for this work. The specific details of the scheme are described in a patent application that is in the submittal process.

In summary, at this stage of the project we had assembled a dedicated and talented team, acquired most of the separator hardware, and assembled it in an appropriately modified laboratory. Several generations of vaporization source were tested, and enough data was generated to point the way toward what ultimately was a successful vapor source. The spectroscopic investigation had successfully identified and demonstrated a workable photoionization pathway, and in doing so, enabled the specification of the required number of process laser wavelengths, the necessary power levels, and the frequency stability requirements.

In the process laser laboratory, the utilities were modified to accommodate the eventual lasers (plumbing in a liquid nitrogen system, for example), and much of the pump laser hardware had been ordered and assembled.

\section{Isoropic Purification of Lead for use in Semiconductor Applications}

During the course of the year, we received several inquiries as to the feasibility of adapting our technology to the isotopic purification of lead. The lead that is used throughout the electronics industries, typically contains small amounts of radioactive ${ }^{210} \mathrm{~Pb}$ (a daughter product of the planet's ubiquitous ${ }^{238} \mathrm{U}$ ) whose ${ }^{210} \mathrm{Po}$ daughter emits an $\alpha$-particle that is known to cause soft errors in electronic circuits (soft errors, where an " 0 " changes to a " 1 " result in an incorrect calculation, a computer crash, etc., but do not result in permanent damage). The ${ }^{210} \mathrm{~Pb}$ is not separable by chemical means, and the possibility existed to pursue an LIS approach to removing the impurity with precisely tuned lasers.

A cursory look at the vapor pressure dependence on temperature showed that lead and thallium were quite similar (see Fig. 6), suggesting that, at least for vapor development purposes, thallium and lead were virtually interchangeable. After receiving all applicable ES\&H training in the proper handling of lead, the source development effort switched from zinc to lead.

Spectroscopically, it is also striking how similar lead and thallium are. The lowest energy transition for both atomic species is ultraviolet ( $283 \mathrm{~nm}$ for lead vs. 378 for thallium). Both transitions have a strong absorption cross section $\left(\sim 2 \times 10^{-12} \mathrm{~cm}^{-2}\right)$ and both have a strongly 
coupled low-lying metastable state. Both first excited states also have a short lifetime, and both have about the same required excitation energy out of the first excited state to the ionization threshold. At the time of our initial interest in lead, the isotope shifts of the first excited states were both known and comparable $(\sim 1 \mathrm{Ghz})$. Finally, the initial photoionization scheme explored for lead made necessary usage of some of the same laser hardware as thallium. Thus, since lead purification appeared so similar to thallium enrichment it was decided to expand the scope of work to encompass lead as well as thallium.

A spectroscopic investigation was launched to measure the unknown and necessary atomic properties for lead. Second, because the first transition falls neatly in the range of a frequency doubled dye laser $(566 \mathrm{~nm})$, the laser development was expanded to include the required dye laser. Third, as mentioned above, the separator development concentrated on lead, both for its own sake as well as a close surrogate for thallium (lead being more environmentally friendly than thallium).

The spectroscopic research yielded all the necessary information required to specify a resonant photoionization pathway, and is embodied in a pending patent application. In addition, the spectroscopy demonstrated, at least in very small scale, the technical feasibility of the pathway, thus demonstrating the proof of concept.

The dye laser development produced a novel and potentially superior dye master oscillator concept for any master oscillator pulsed amplifier laser system, as is required by the Uranium AVLIS Program. Most of the effort in this area was spent building, testing, and characterizing this concept.

\section{Separator System Development}

With the expansion of the scope of work came some additional resources that allowed us to dedicate some people to various development tasks. Relatively rapid progress was made in the separator development area. Fig. 7 shows a typical configuration of separator components. They include a vaporizer for producing an atomic vapor, a collimator to restrict the vapor flow between the extractors and to limit the Doppler width, an extractor that electrostatically removes the photoions that are produced by the lasers from the vapor stream, and a collector where the remaining vapor condenses. A new concept for the thermal source vaporizer was designed and built. When tested in the separator system it was found to give the required atomic vapor properties.

The first requirement, of course, is that the vapor be an atomic vapor. Our previous sources showed evidence of aerosol behavior. Second, the vapor is required to undergo a supersonic expansion in order to become a cold, collisionless vapor. Conceptually, LIS works by dividing the input vapor into two separate streams, one that is enriched in the photoionized species, and the other stream that is depleted. The reason for the collisionless requirement is that collisions lead to mixing of the enriched and the depleted streams. The requirement for the vapor to be cold, i.e. for most of the atomic population to be in the lowest electronic state of the atom, 
is to make it accessible to the photoionization pathway (which only accesses atoms only in their lowest electronic state) as much of the vapor as possible.

A series of experiments using this vapor source were conducted to characterize the throughput, vapor velocity, and the spatial distribution of the vapor versus the source temperature. The directionality of the flow and the throughput demonstrated were consistent with the throughput requirements of the market for thallium. Furthermore, by the appropriate choice of vapor aperture we were able to reduce the Doppler width of the vapor to less than the isotope shift between neighboring isotopes, in this case between one of the hyperfine components of ${ }^{207} \mathrm{~Pb}$ and ${ }^{210} \mathrm{~Pb}$.

A thermal vaporizer structure capable of operation at $1100^{\circ} \mathrm{C}$ was constructed. A slot was built into the lid for the vapor to expand through. The purpose of using a slot, as opposed to a point, was to make the source more like a line source than a point source. The advantages of a line source are two fold. First, expansion through a slot produces a vapor that is colder in the short axis than in the long axis. Along the long axis, however, the Doppler width due to the geometrical expansion is smaller. After the vapor passes through the collimator with the long axis of the slot oriented parallel to the long axis of the collinator, the combined (thermal and geometrical) Doppler width of the vapor was narrower than in the short axis. The other advantage of the slotted vaporizer over point source is that, since the area is greater for the slot than for the point, and since the vaporization rate is proportional to the area, the throughput is greater for the slot than for a point. The successful demonstration of this vaporizer culminated an intensive one year effort, and remains one of the major achievements of the project. This same source, usable for both thallium and lead, is applicable to a variety of other potentially viable elements including calcium, strontium, and barium.

After developing the vapor source, a collimator was then needed. The purpose of a collimator is to restrict or eliminate the semispherical distribution typically produced by a vaporizer to a much smaller region of space. Here again, our first concept was only partially successful. Through use of a laser diagnostic (see discussion below) to probe the Doppler distribution of the vapor, and also by the analysis of mass accumulated on a variety of tabs judiciously placed throughout the chamber, we were able to determine that the combination of the source slot and the collimator were restricting the vapor flow appropriately: High mach numbers characterized the vapor flow.

\section{Laser Diagnostic Experiments for Thallium}

At this point in the development, we were able to integrate the first thallium process wavelength (see discussion below) with the separator vacuum chamber. We took advantage of the process wavelength to look at the vapor profile, i.e. the Doppler width, velocity, and angular distribution. The first test involved covering the collimator with a solid plate with three slits cut into it: One directly above the source and two at each end of the collimator length. The absorption profile for such a configuration yields three peaks in frequency space. The width of the peaks is proportional to the Doppler width of the vapor, the separation of the peaks gives 
information about the flow velocity, and the relative heights of the peaks is a measure of the angular density distribution along the collimator. Fig. 8 shows a typical absorption scan and a best-fit to the data. The conclusions deduced from this experiment were used to determine laser and source requirements.

A second diagnostic test measured the absorption profile along the entire path of the collimator. Fig. 9 shows an absorption scan and the resultant best fit. In this experiment, the source temperature was varied over a range of $200^{\circ} \mathrm{C}$ and the corresponding line-averaged density was measured from the laser absorption. The results of this study were used to characterize a simple two-dimentional vapor model for the thermal spatial variation (see Fig. 10) that, in turn, was used to determine source operating conditions as a function of vapor throughput.

\section{The Separator Extractor Sub System}

The next effort in the separator system was to develop and demonstrate the electrostatic extractors. The extractor power supply architecture and operation is proprietary information and will not be described here, but the devlopment effort was involved, and ultimately successful.

The extractor plates were straightforward - stainless steel structures to which voltage could be applied. Stainless steel was chosen because of the care with which thallium can be removed using acid. The dimensions of the extractors were both consistent with the vacuum chamber, the vapor properties (particularly the flow velocity) after the collimator, and the laser beam dimensions. Lastly, the collector, a sheet of stainless steel, was placed above the extractors centered over the collimator opening.

The entire separator system was tested in a number of vaporization runs to verify the electrical integrity of the extractors in the presence of the vapor, and to quantitatively measure the distribution of the condensed vapor on the internal structures of the separator hardware. Ultimately, the separator system was found to be sufficiently developed to support enrichment.

\section{Laser System Development}

The laser system development proved to be very challenging, especially with the resources available for the project. All of the process colors (combinations Nd:YAG-pumped of dye and Ti:S) need to be temporally and spatially overlapped. In order to save costs, and to more efficiently utilize our limited resources, some of the wavelengths were generated through coamplification. The process lasers also need to be modulated in frequency in a specific manner in order to span the Doppler profile of the vapor. Without this modulation, only a very small portion of the vapor plume is in resonance with the laser light, and therefore, only a small amount of the available isotopes could be ionized. In addition, the frequency stability requirements for both thallium and lead were predicted (using in-house first- principal photoionization codes) to be $<50 \mathrm{MHz}$. This requirement forced us to develop only single mode lasers. Also, since the first excitation step for both thallium and lead require frequency doubling of Ti:S, and since the efficiency of the second harmonic generation process is very sensitive to beam quality, near diffraction-limited fundamental laser radiation was required. Finally, since the separator system 
was relatively far away from the process lasers, the laser delivery system required fairly stringent pointing and centering loops to keep the laser beams aligned spatially.

All of these issues were worked through throughout the course of the project. As with most any laser development effort, progress was always generously interspersed with setbacks: from burnt optics, vendor problems (quality control) with power supplies for seed lasers resulting in destruction of one of a kind hardware, cooling water lines and flooring attached to the laser monoliths designed for frequency stability, and frequency doubling crystals cut incorrectly to loss of key personnel and other critical resources. Nevertheless, significant, and sometimes remarkable, progress was made.

The final system was small, compact, and once aligned and functioning, performed reasonably well. Listed in the table below are some of the system performance parameters.

Table 1: Laser System Performance Parameters

\begin{tabular}{|l|c|}
\hline \multicolumn{1}{|c|}{ Parameter } & Value \\
\hline Frequency Stability & $\pm 20 \mathrm{MHz}$ \\
\hline Operational Run Time ${ }^{\mathrm{a}}$ & $\sim 100 \mathrm{~h}$ \\
\hline Pulse Duration (FWHM) & $60 \mathrm{~ns}$ \\
\hline Pulse Repetition Frequency & $5-30 \mathrm{KHz}$ \\
\hline Beam Quality & $<1.5 \mathrm{XDL}$ \\
\hline Maximum Output @ 829 nm (Single Amplifier) & $45 \mathrm{~W}$ \\
\hline Doubled Single Amplifier Output @ $378 \mathrm{~nm}^{\mathrm{b}}$ & $1 \mathrm{~W}$ \\
\hline Wavelength Range Tested & $700 \mathrm{~nm}-900 \mathrm{~nm}$ \\
\hline
\end{tabular}

a. Limited by staffing, and no attempt to harden the operational design was made.

b. Other wavelengths were amplified through two stages.

\section{Integrated Operations}

By the completion of this project almost all of the major aspects of laser isotope separation had been demonstrated, except for the planned series of enrichment demonstration experiments. The groundwork for enrichment, however, had been laid. The technical framework for the description of the efficiency of LIS is captured in a "Point Design".

A Point Design is a specification of all the required operating parameters of the process, for example, the extractor spacing, voltage, materials, minimum and maximum voltages and timing, the laser powers and wavelengths, the source rates, the number of external stages, etc. Ultimately, this information is used to quantify the efficiency of the process, both technical and economical. Over the course of the project, a Point Design for thallium and lead were developed, both proprietary in their details, and expressed in patent applications.

The technical enrichment performance of an LIS process can be characterized by two

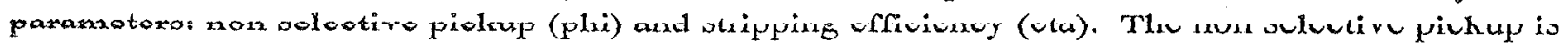


the fraction of the throughput that is collected in the product stream that is at natural assay whereas the stripping efficiency is the fraction of the isotope of interest in the feed material that is removed from the feed stream and collected as product.

Clearly, at least two parameters are required for the specification of a separation process. For example, having a low or high value of the stripping efficiency provides no complete description of the amount nor assay of the product material. However, for an application such as purification (the selective removal of an impurity), the stripping efficiency is particularly important since it is sufficient to characterize the assay of the purified stream. Similarly, the non selective pickup provides no information about the assay of the purified (i.e. depleted) stream but is required in order to (partially) characterize the quantity of material going to the enriched and depleted streams. The definitions of eta $(\eta)$ and phi $(\varphi)$ are shown below:

$$
\begin{aligned}
& \eta=\frac{X_{F}-X_{D}}{X_{F}\left(1-X_{D}\right)} \text { and } \\
& \varphi=\frac{\left(1-X_{E}\right) E}{\left(1-X_{F}\right) F}
\end{aligned}
$$

where $X_{F}, X_{D}$, and $X_{E}$ are the assays for the feed material, the depleted stream, and the enriched stream, respectively.

Traditional enrichment stages are characterized by two parameters, the separation factor $(\alpha)$ and the cut $(\theta)$, defined as:

$$
\begin{aligned}
& \alpha=\frac{X_{E} /\left(1-X_{E}\right)}{X_{D} /\left(1-X_{D}\right)} \text { and } \\
& \theta=\frac{E}{F}
\end{aligned}
$$

where $\mathrm{E}$ is the total Enriched stream and $\mathrm{F}$ is the total Feed stream.

In terms of $\eta$ and $\varphi$ :

$$
\begin{aligned}
& \alpha=\frac{\varphi+(1-\varphi) \eta}{\varphi(1-\eta)} \text { and } \\
& \theta=\varphi+(1-\varphi) \eta X_{F} .
\end{aligned}
$$

For traditional isotope separation techniques such as gaseous diffusion or centrifuges, the separation factor is usually about $\alpha=1.001$, that is, the enrichment is only a small increment. For an LIS process, values of the separation factor tend to be in the range of 6 to 50 , whereas the cut tends to be 2 to $40 \%$. It is because of the relatively high separation factors that the economics of LIS can be favorable for applications that are large enough to justify the R\&D. 
Available to us throughout the development effort were key personnel of the AVLIS Program (uranium laser isotope separation) and their suite of detailed process physics codes. These were exercised liberally to arrive at the detailed specification of the likely operating point for both lead and thallium, if either of these applications were to be taken into production.

In summary, at the end of the project the enrichment performance looked promising based on the process models (AVLIS models appropriately modified as well as those developed inhouse). Economics models were developed that added the anticipated capital costs of all the process equipment, and operational costs in order to anticipate likely internal rates of return if either of the projects are taken through to production. The separator effort had developed and tested all components individually and integrated into the separator system. The laser system developed all the required process laser wavelengths that also met all of the requirements of beam quality, spectral purity, modulation format, etc., except for the desired amount of laser power in some of the systems (there was enough power available for an enrichment demonstration, had we had more time). In short, the project is well poised for a convincing demonstration for either of these isotopes, and potentially several isotopes that are within a similar wavelength range.

\section{Conclusions and Future Work}

We have completed all of the fundamental development work required for a demonstration of LIS of at least two isotopes of potential market interest. These developments include the preparation/adaptation of a facility, development and demonstration of a complete separator system, including source, collimator, extractors (enriched material collector), and depleted material collector, and a multi-colored laser system, complete with pointing and centering loops, wavelength control, and laser delivery system. In addition, the laser development also made possible, for the first time, high power, high pulse repetition frequency laser systems in the $280 \mathrm{~nm}-450 \mathrm{~nm}$ wavelength range. The specific technology developed for ${ }^{210} \mathrm{~Pb}$ are largely applicable to other medical and industrial isotopes used in large quantities, e.g. strontium, barium, calcium, and a variety of the lanthanide elements. The laser systems (and components thereof) will be applicable to a wide range of other industrial and/or health-critical isotopes of immediate interest.

Three additional steps must be taken to demonstrate and deploy the technology. First, significant industrial support for continuing the project must be secured in the form of a "Work for Others" (or other) arrangement or by purchasing a futures contracts (or the like). Second, a credible enrichment or purification demonstration to produce $>100$ grams of a specific enriched or purified isotope must be completed, thus validating the enrichment performance models and the hardware configuration. After these steps, the last step is to develop a business plan that schedules and resource loads the remaining development and deployment of a production system. Such a production system could significantly increase the nations's capacity of commercially significant isotope production at sharply reduced prices. A facility housing this production system could grow into a multisotope production facility supported by annual revenues greater than $\$ 10 \mathrm{M} / \mathrm{y}$. 


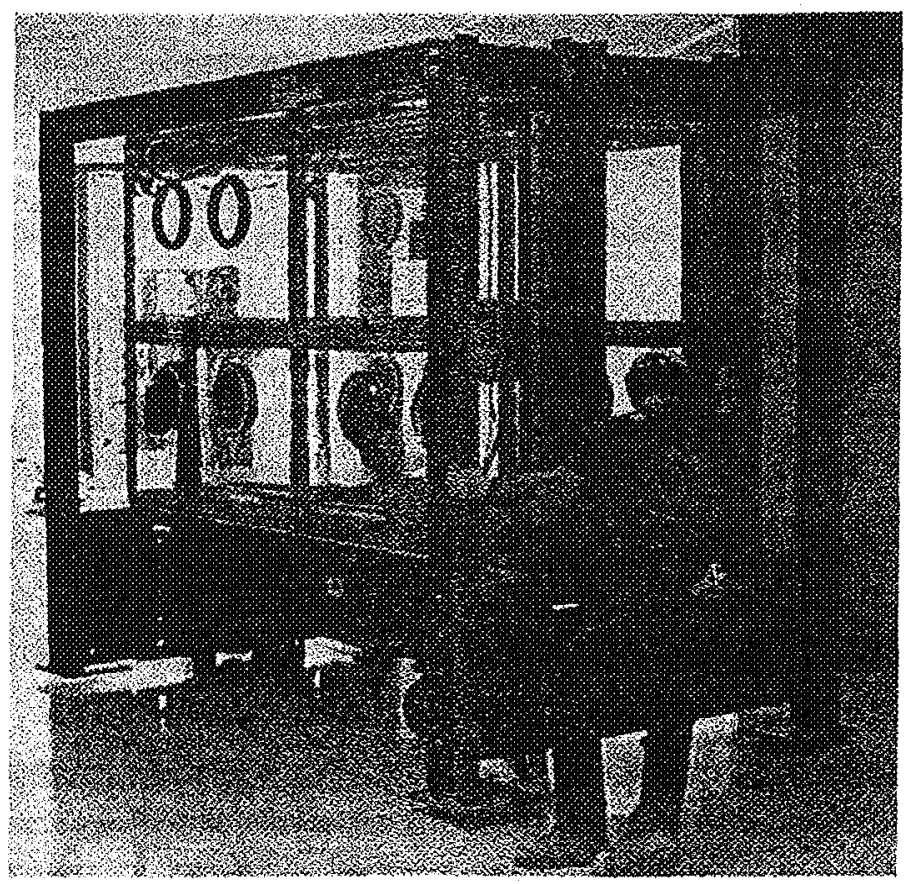

Figure 1(a)

Separator hardware was identified and moved into the facility. The glovebox, which the separator vacuum chamber rests upon, is shown above being moved into the facility.

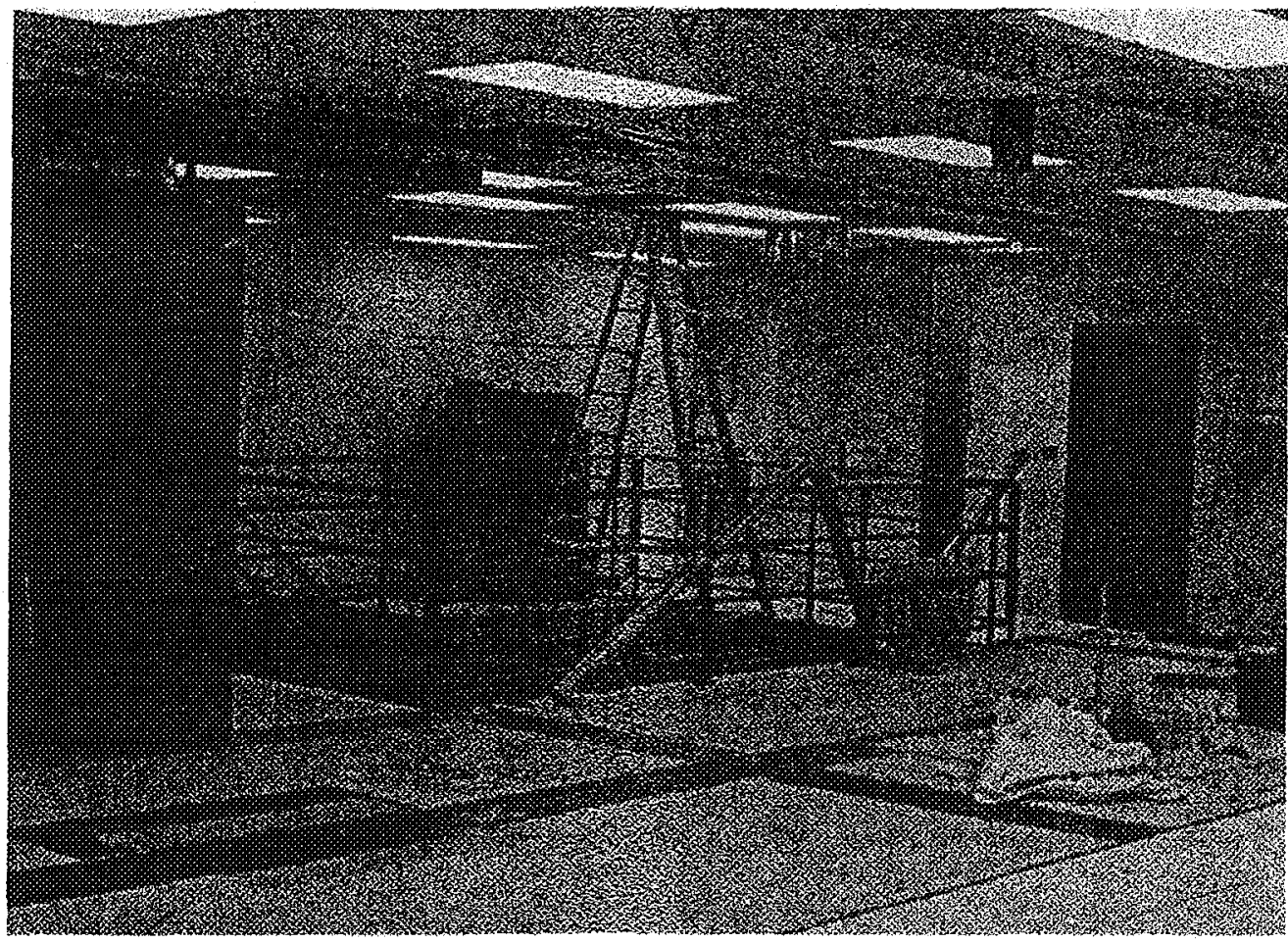

Figure 1(b)

The glovebox was placed into a pit, shown above, to allow easier access to the separator vacuum chamber. On the left side of the figure, the computer control racks can be seen, and the door to the adjoining laser laboratory can be seen to the right. 


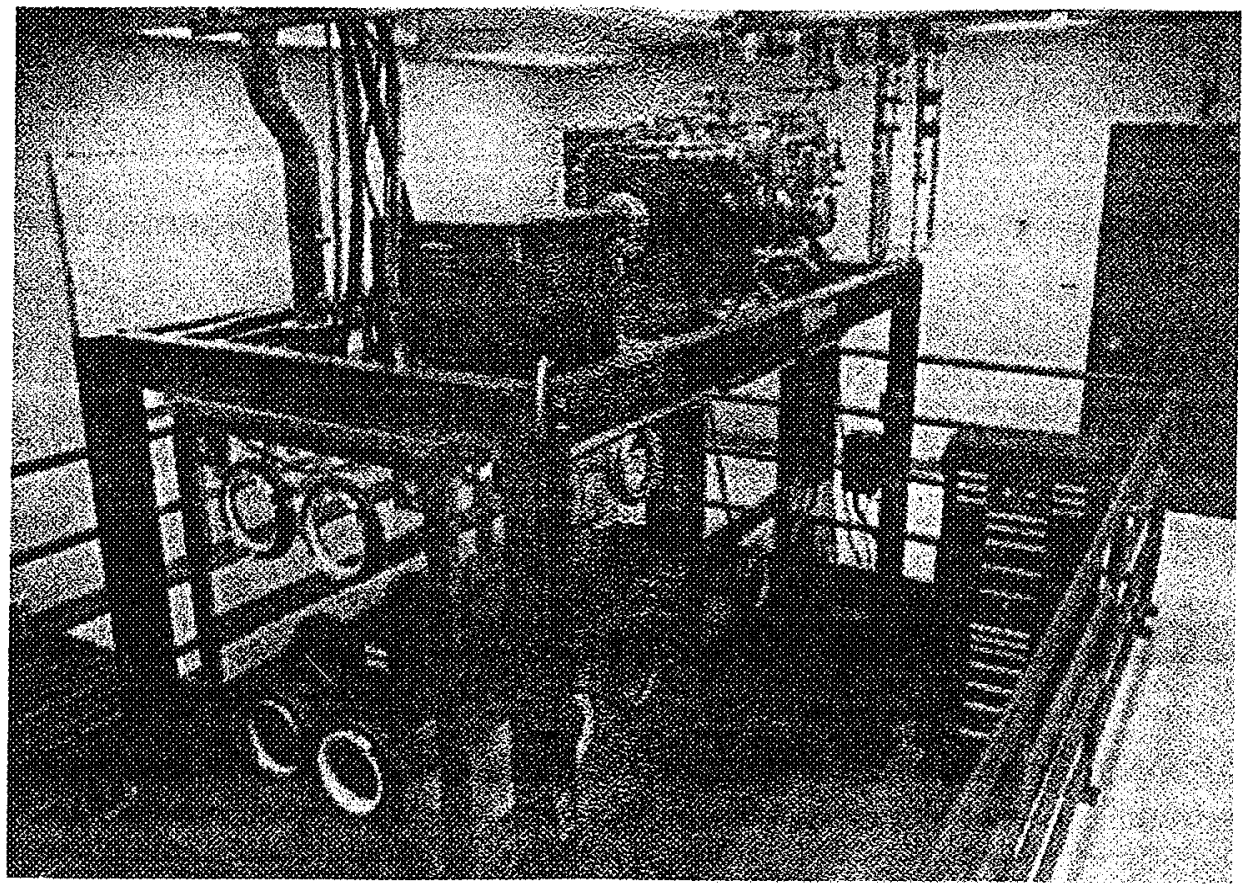

Figure l(c)

The glovebox with the separator inside the pit is shown above. The separator is on the far side, on top of the glovebox. The inner structures of the separator (source, collimator, extractors, and collector) are installed onto a base plate inside the glovebox and then raised into position, scaling the separator vacuum chamber using turn screws at the far end of the glovebox.

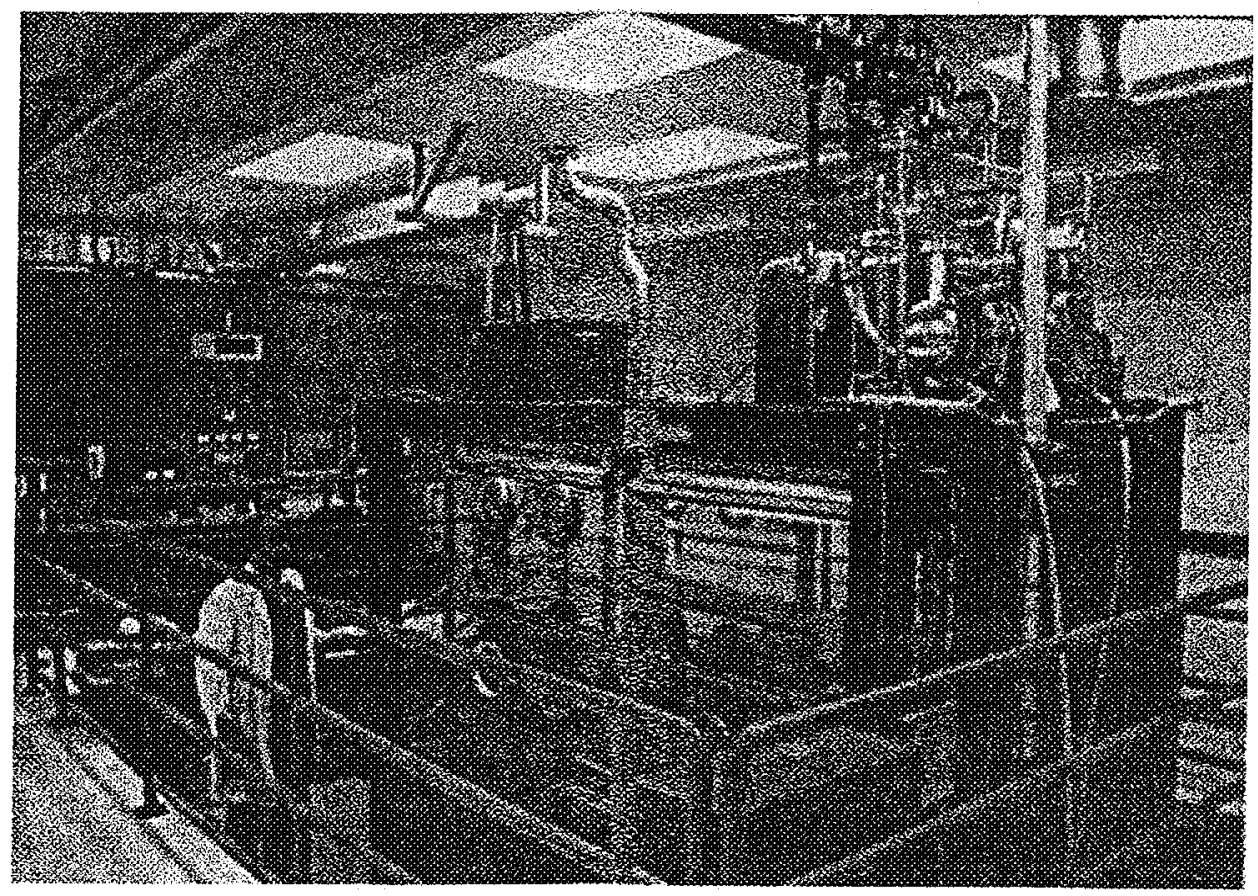

Figure $1(d)$

Another view of the separator assembly, with the computer controls in the background, is shown in this figure. All of the plumbing. electrical, and laser access narts can also he seen 


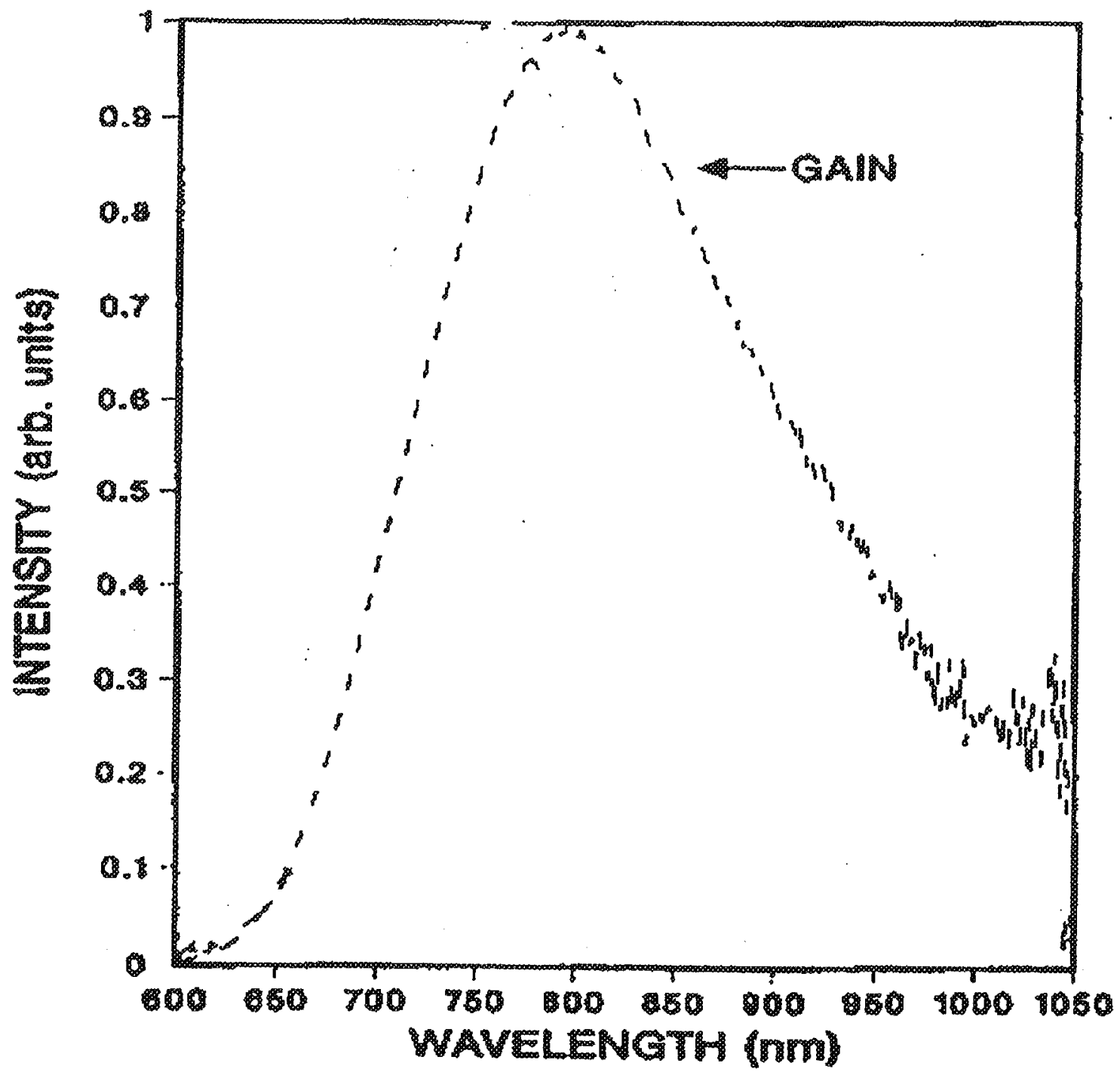

Figure 2

Ti-sapphire gain line shape*

(*) P. Moulton, J. Opt. Soc. Am., Volume 3, No. 1, p126, Jan. '86. 


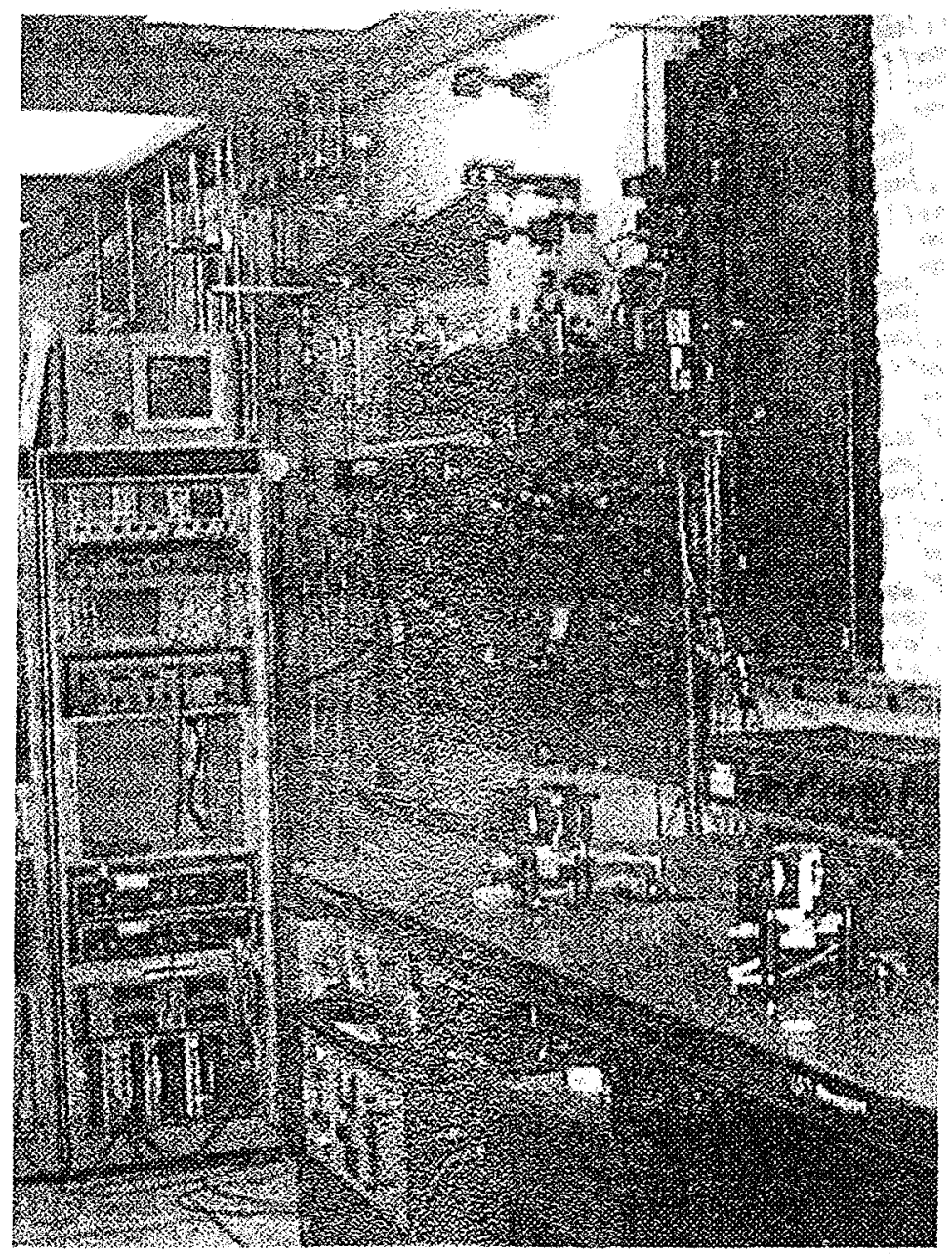

Figure 3

A view of the MLIS laser hall showing on the horizontal table in the foreground two cryrogenically cooled Ti-S amplifier cells with green YAG pump Mirrors installed and one of the four 40W green pump YAGs. One $10 \mathrm{~W}$ green $\mathrm{YAG}$ and the seeded TSO system are mounted vertically on the wall behind the amplifiers. 


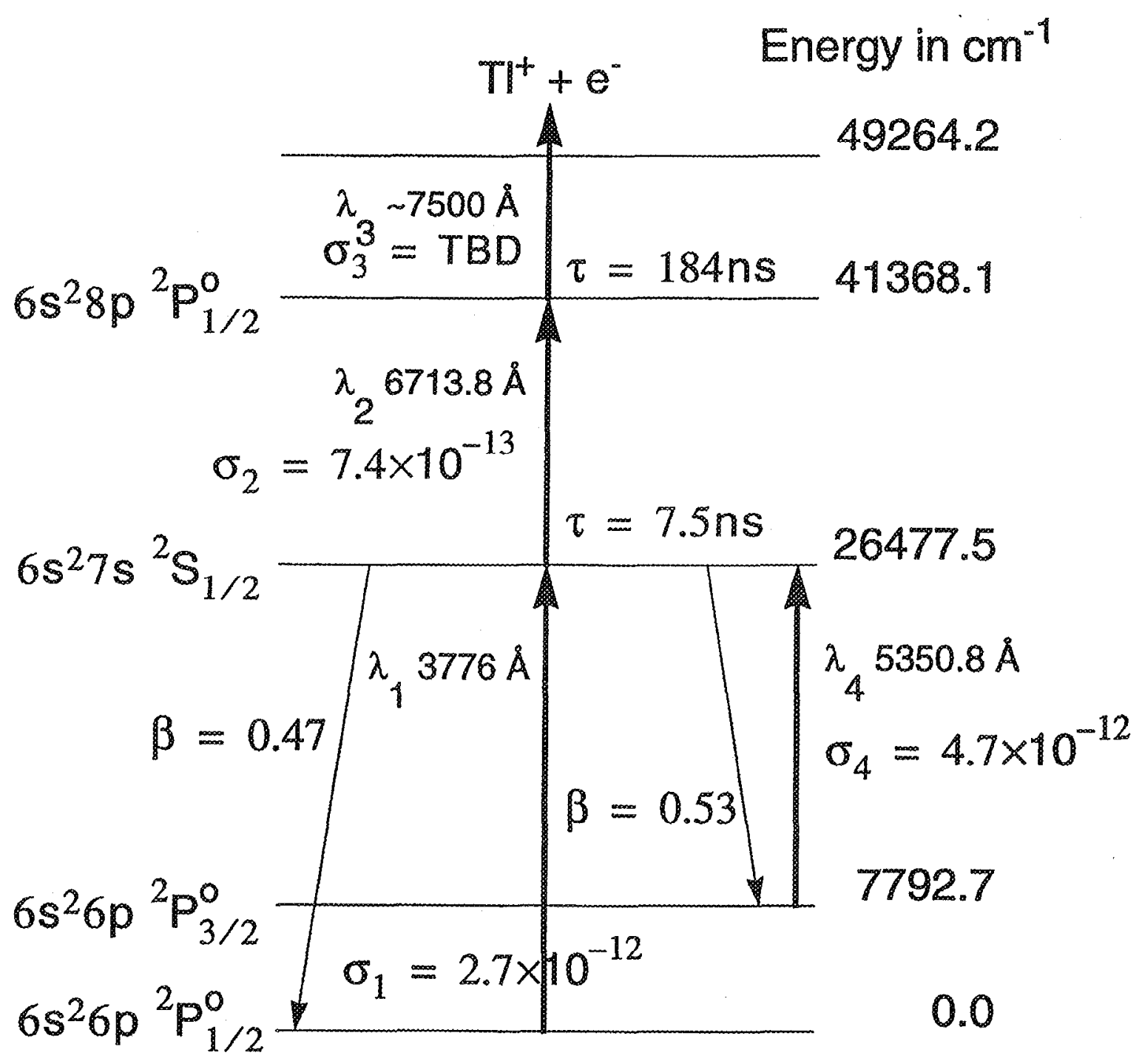

Figure 4

Thallium spectroscopy - what had been known at the start of the project. Several alternative photoionization pathways were explored, and the unknown cross sections, hyperfine splittings, and isotope shifts were measured as part of the project. 


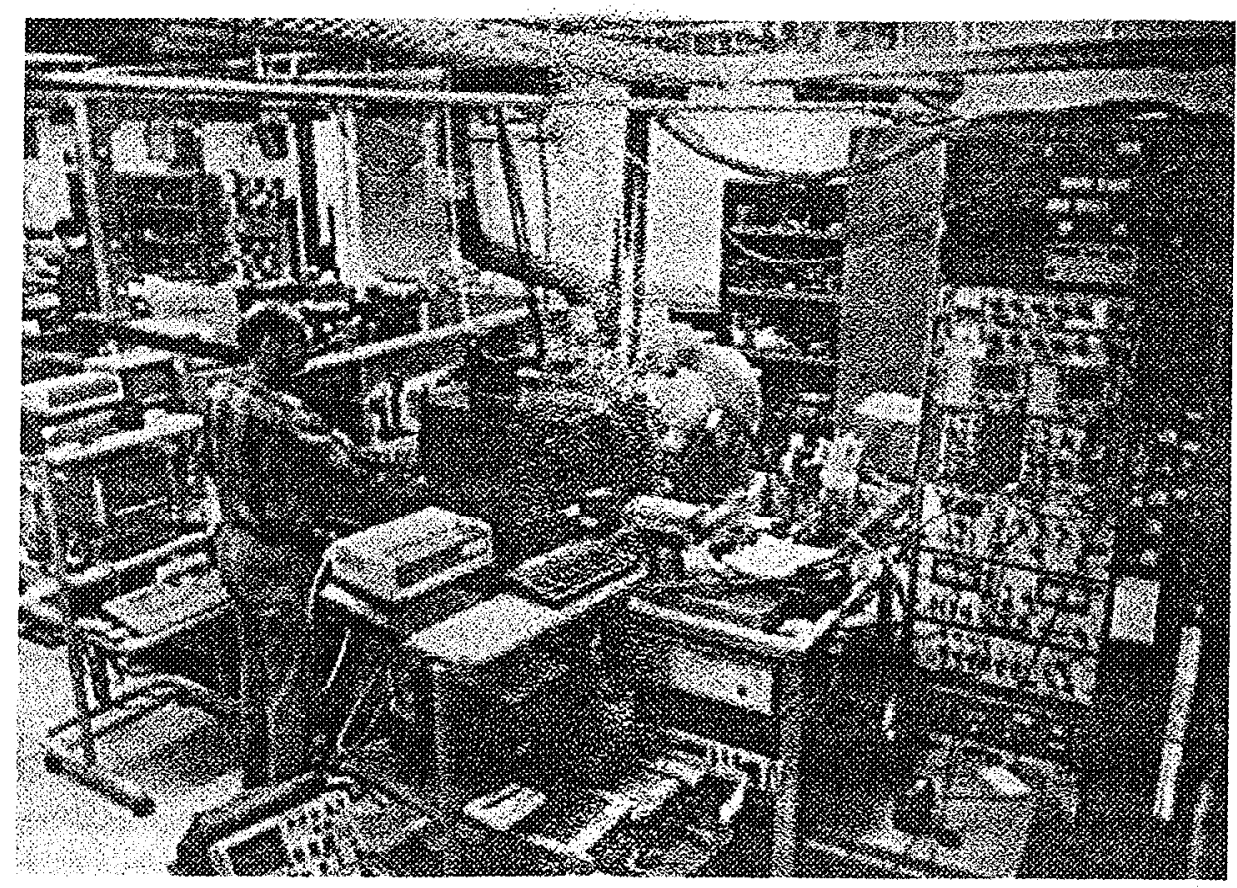

Figure 5(a)

Shown above is the spectroscopy laboratory used to make the required photoionization measurements. Various diagnostics can be seen, as well as the photoioniztion chamber.

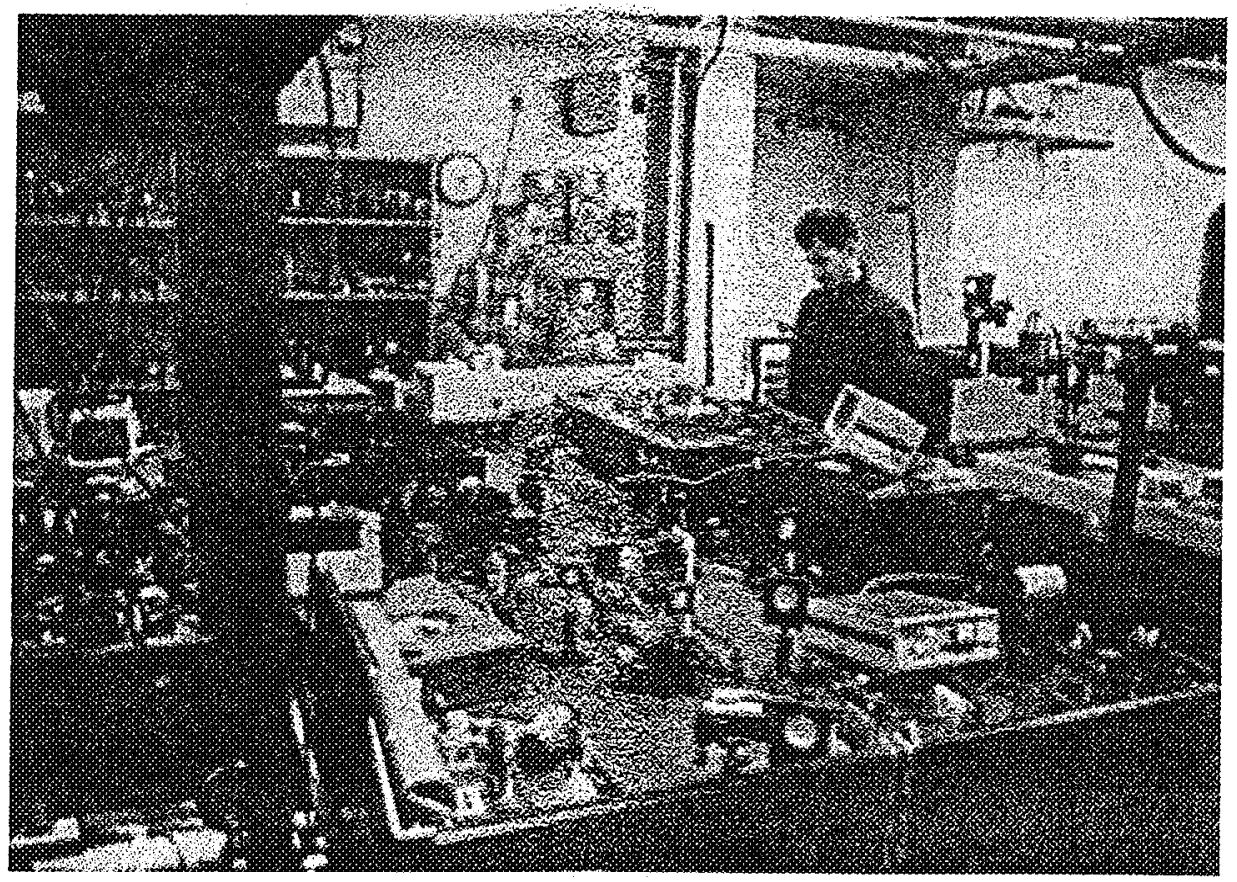

Figure 5(b)

Another view of the spectroscopy laboratory. A variety of pulsed dye lasers, ning dye lasers, titanium sapphire lasers, argon ion lasers, and Krypton ion lasers were used to take the necessary illeasur enents. 


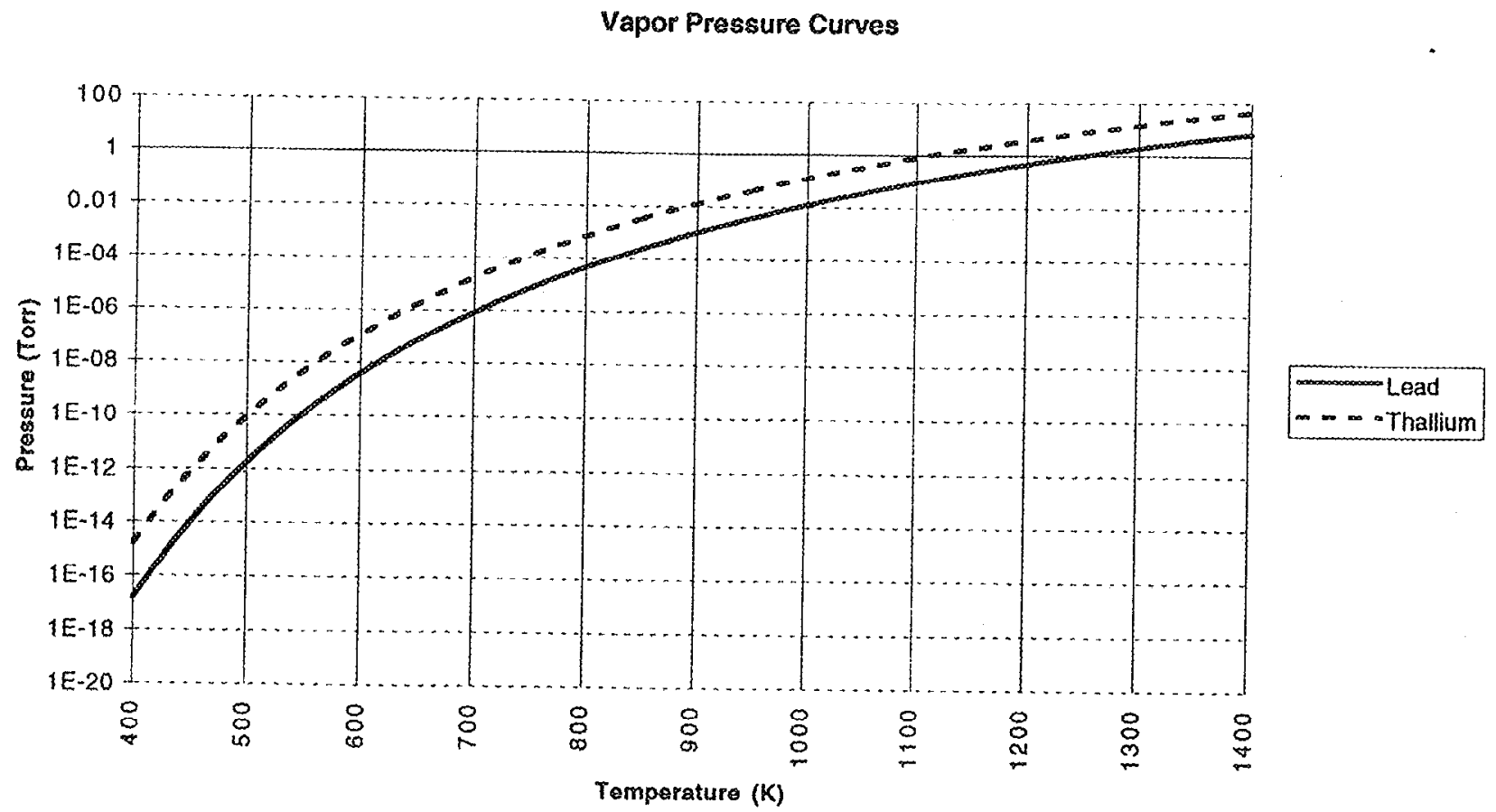

Figure 6

Vapor pressure versus temperature for both elemental thallium and lead. The operating range required for reasonable throughputs is from $1100 \mathrm{~K}$ to $1400 \mathrm{~K}$.

Vacuum Chamber

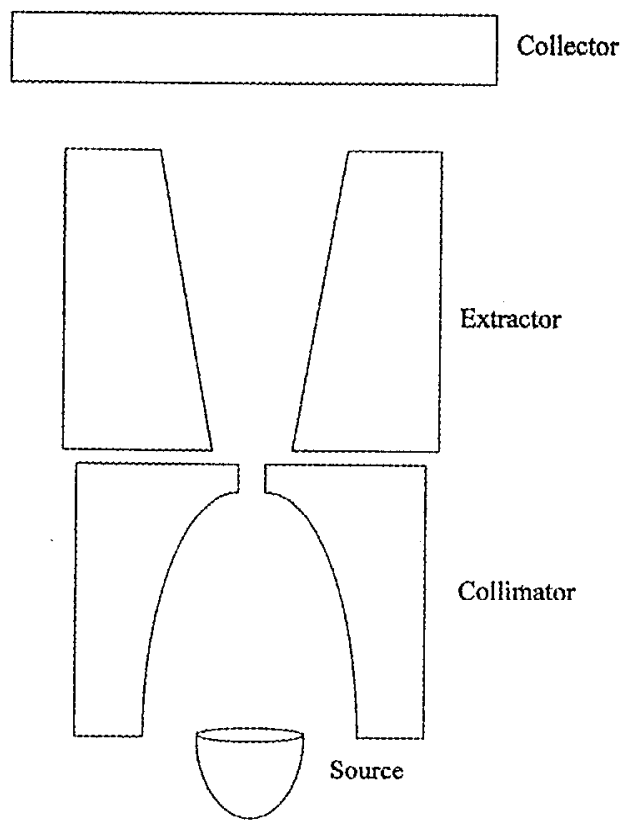

Figure 7

Major components of the complete separator svstem (see text). The nrocess lasers are iniented in between the extractors, through optical grade vacuum windows. 


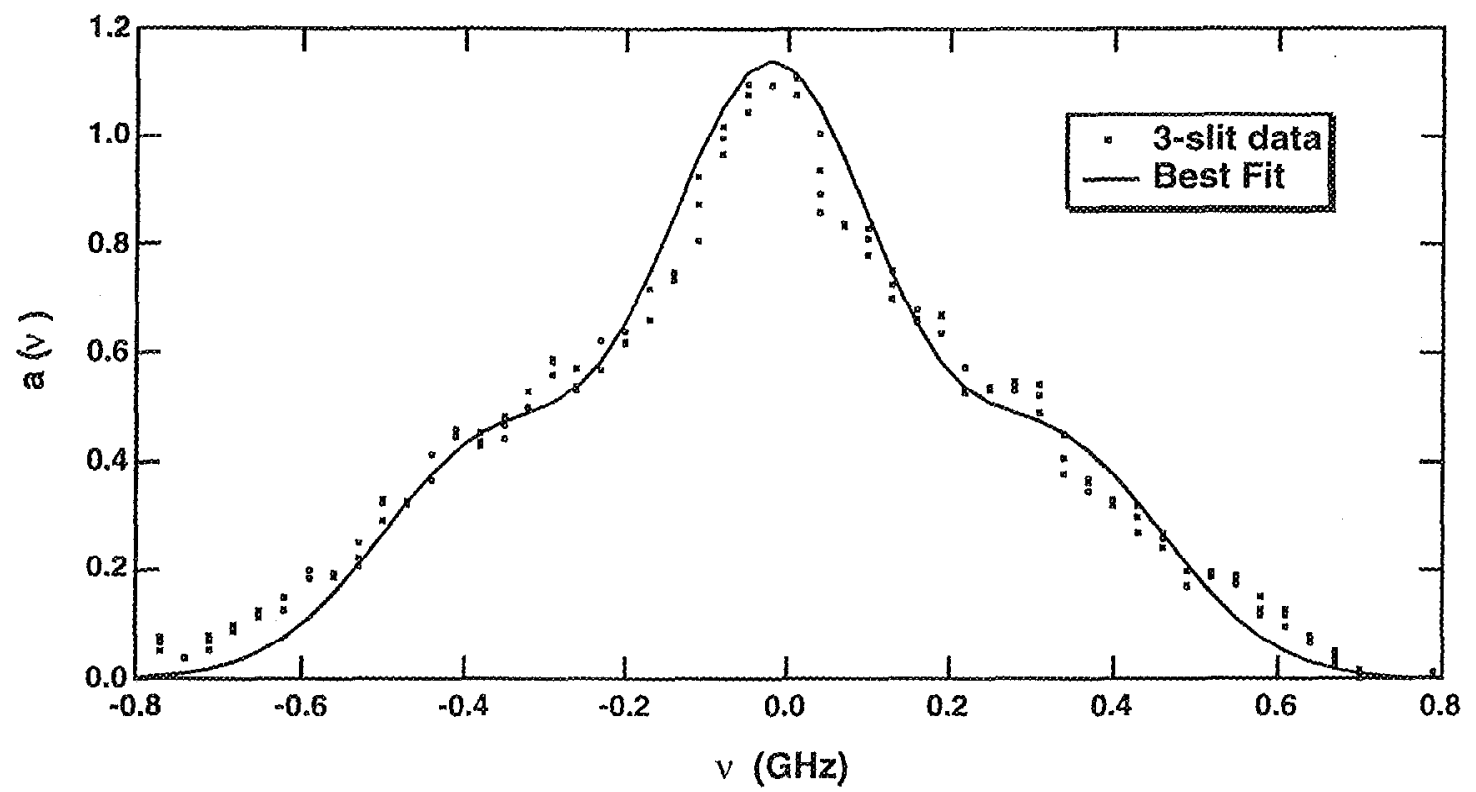

Figure 8

Absorption profile (dots) and best fit (curve) for three-slit vapor properties measurement.

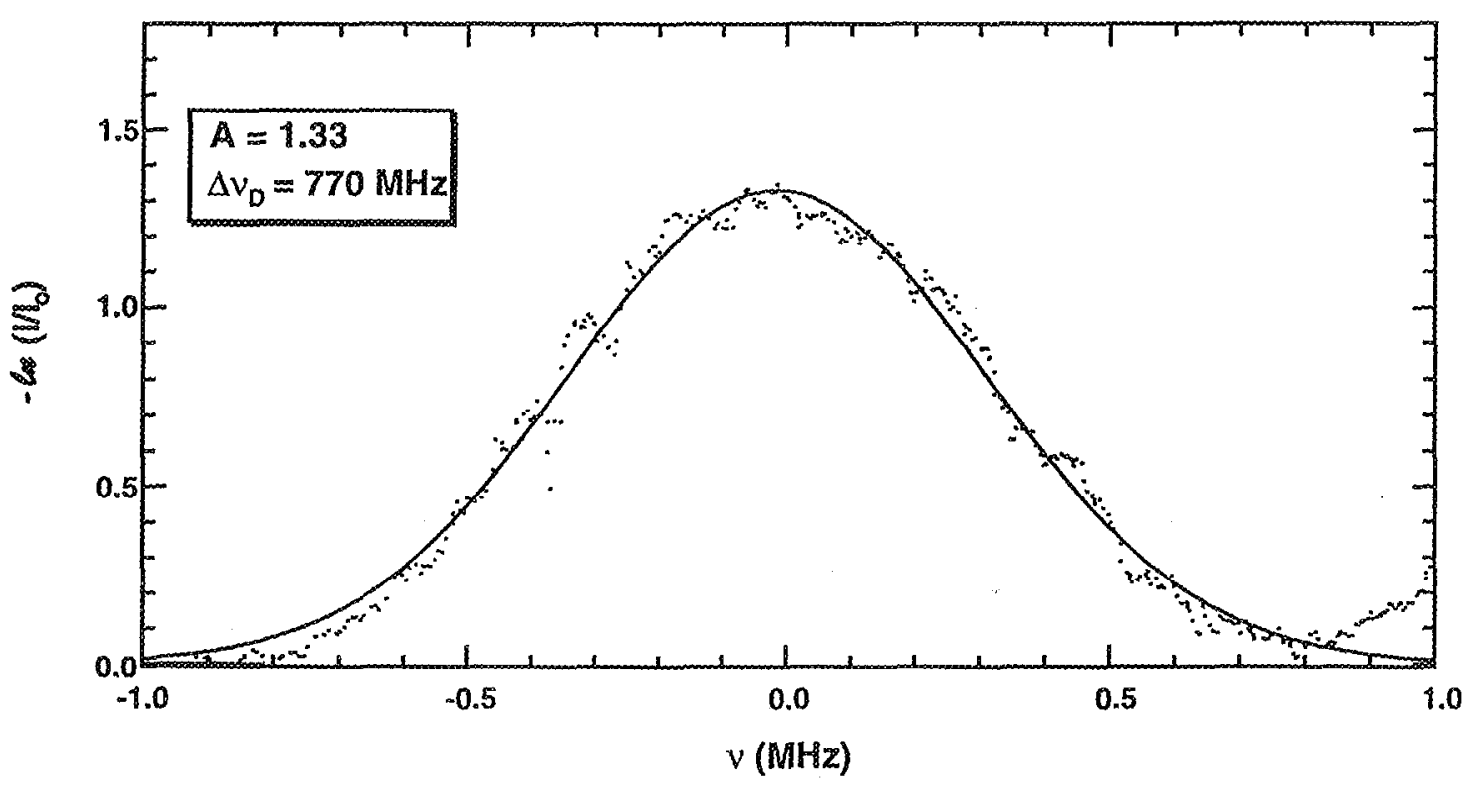

Figure 9

Absorption profile (dots) and best to fit data (curve) along entire collimator length. 


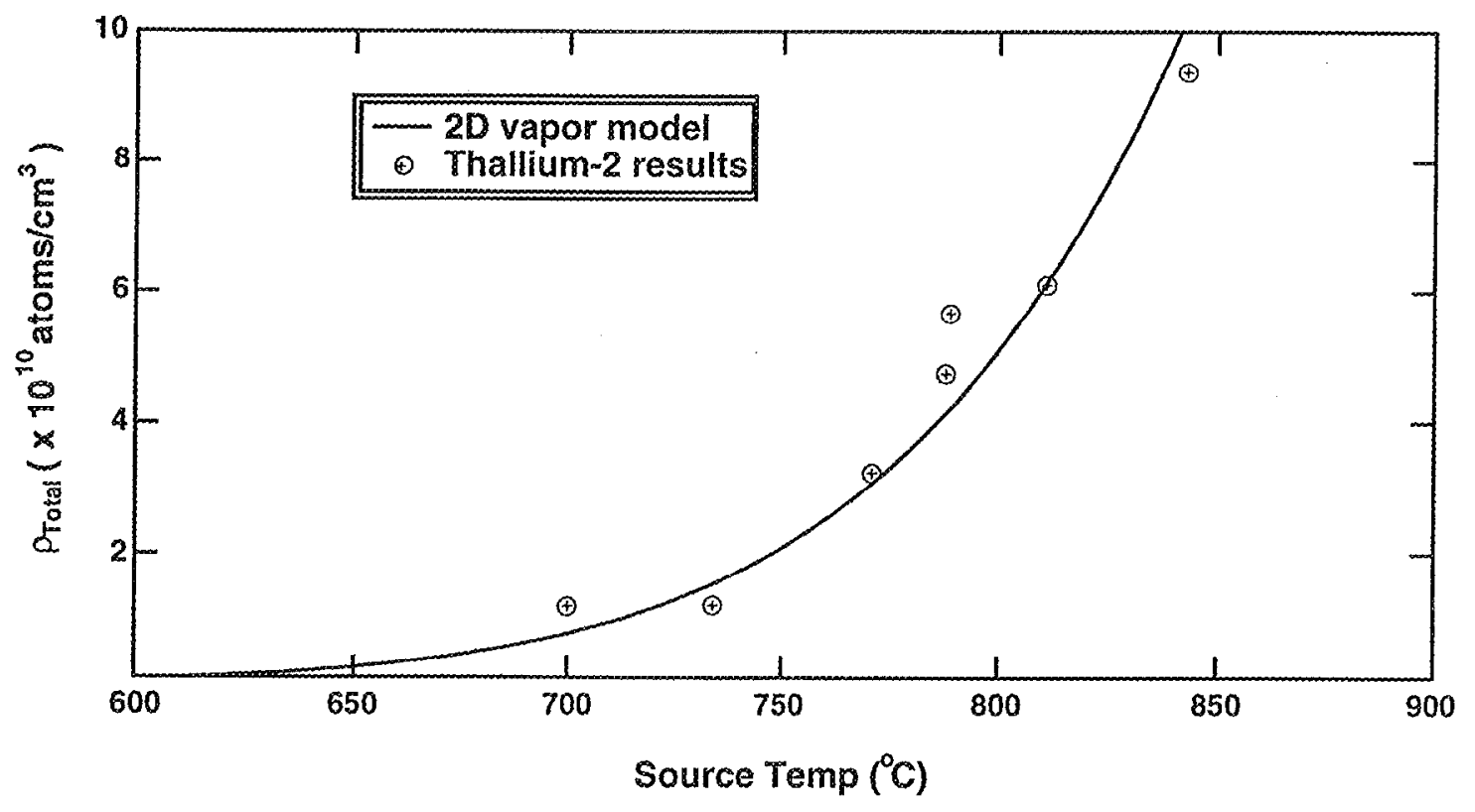

Figure 10

Comparison of measured (circles) to predicted (curve) density as a function of source temperature. The predicted curve is from a simple two-dimensional vapor expansion model. 